Bull. Korean Math. Soc. 52 (2015), No. 4, pp. 1185-1199

http://dx.doi.org/10.4134/BKMS.2015.52.4.1185

\title{
OPTIMAL CONTROL OF THE VISCOUS WEAKLY DISPERSIVE BENJAMIN-BONA-MAHONY EQUATION
}

\author{
LeI ZHANG AND Bin LiU
}

\begin{abstract}
This paper is concerned with the optimal control problem for the viscous weakly dispersive Benjamin-Bona-Mahony (BBM) equation. We prove the existence and uniqueness of weak solution to the equation. The optimal control problem for the viscous weakly dispersive BBM equation is introduced, and then the existence of optimal control to the problem is proved.
\end{abstract}

\section{Introduction}

It is well known that the Benjamin-Bona-Mahony (BBM) equation

$$
u_{t}-u_{x x t}+u_{x}+u u_{x}=0
$$

was initiated by Benjamin, Bona, Mahony in [2], which is motivated by the Korteweg-de Vries (KdV) equation [4]

$$
u_{t}+u_{x x x}+u_{x}+u u_{x}=0
$$

for modeling water waves of small amplitude and large wavelength. In all these equations, $u$ denotes a wave amplitude or velocity, $x$ is proportional to the physical distance and $t$ is proportional to the elapsed time.

When the non-linear terms of Eq.(1.1) are replaced by $u^{p} u_{x}$ for $p>0$, the resulting equations reads

$$
u_{t}-u_{x x t}+u_{x}+u^{p} u_{x}=0,
$$

which is called a generalized BBM equation. It has been found that (1.1) possesses global solutions for smooth initial data [2] for $p=1$, which can be easily extended to the general case (1.3), and the solitary wave solutions of (1.3) are stable when $p<4$ [2].

A great deal of research has been devoted to the BBM equation. For example, Avrin and Goldstein [1] studied the global existence for the BBM equation

\footnotetext{
Received July 20, 2014.

2010 Mathematics Subject Classification. 35D40, 35Q53, 49J20, 49L25, 93C20.

Key words and phrases. viscous weakly dispersive, BBM equation, existence, optimal control.

This work was partially supported by NNSF of China (Grant No. 11171122).
} 
in arbitrary dimensions. Chen [3] discussed the periodic initial-value problem for BBM equation. Nickel [8] obtained the elliptic solutions to a generalized BBM equation. Zeng [18] obtained the existence and stability of solitary wave solutions of equations of BBM type. Moreover, we also remark that some studies are concerned with the control of BBM equation, such as [7, 9]. In [9], Rosier and Zhang studied the unique continuation property and control for the BBM equation on a periodic domain. And in [7], Micu discussed the controllability of the linearized BBM equation.

On the other hand, many results have also been obtained for the optimal control of partial differential equations, we refer to [6, 10] and references therein. Specially, here it is worth mentioning about the optimal control problems for the viscous weakly dispersive equations. For example, Shen and Gao [11] discussed the optimal control of the viscous weakly dispersive Degasperis-Procesi equation. Vedantham [17] studied the optimal control of the viscous Burgers equation using an equivalent index method. Sun [13] obtained the maximum principle for optimal distributed control of the viscous Dullin-Gottwald-Holm equation. Tian [16] considered the optimal control of the viscous Camassa-Holm equation. Lenells and Wunsch [5] discussed the weakly dissipative Camassa-Holm, Degasperis-Procesi and Novikov equations. Smaoui [12] studied the boundary and distributed control of the viscous Burgers equation. L. Tian and C. Shen [15] considered the optimal control of the viscous Degasperis-Procesi equation.

To the best of our knowledge, few papers can be found in the literature for the optimal control problem of the viscous weakly dispersive BBM equation. So, being inspired by the above mentioned, the purpose of this paper is to study the optimal control problem for the following viscous weakly dispersive BBM equations

$$
u_{t}-u_{x x t}-\varepsilon\left(u-u_{x x}\right)_{x x}+u_{x}+u u_{x}+\lambda\left(u-u_{x x}\right)_{x}=0,
$$

where $\lambda\left(u-u_{x x}\right)_{x}$ is a dispersive item, $\varepsilon\left(u-u_{x x}\right)_{x x}$ is a viscous item, $\varepsilon>0$ and $\lambda>0$ are two constants. More specifically, we are concerned with the following control system

$$
\left\{\begin{array}{l}
\min \{J(y, \bar{\omega})\}=\frac{1}{2}\|C y-z\|_{S}^{2}+\frac{\delta}{2}\|\bar{\omega}\|_{L^{2}\left(Q_{0}\right)}^{2} \\
y_{t}-\varepsilon y_{x x}+\lambda y_{x}+u_{x} y+u_{x}-u_{x} u_{x x}=f+B^{*} \bar{\omega} \\
y(x, 0)=u(x, 0)-u_{x x}(x, 0)=\psi \in H \\
u(0, t)=u(1, t)=u_{x}(0, t)=u_{x}(1, t)=u_{x x}(0, t)=u_{x x}(1, t)=0
\end{array}\right.
$$

where $y=u_{x}-u_{x x}$. The aim is to match the given desired state $z$ by adjusting the body force, $\bar{\omega}$ is a control belongs to the Hilbert space $L^{2}\left(Q_{0}\right)$ with minimal energy and work, the first term in cost functional measures the physical objective, the second one is the size of the control, where $\delta>0$ plays the role of a weight. 
The paper is organized as follows: In Section 2, we obtain the existence and uniqueness of weak solutions to (1.5), and establish the inequality for the norm of weak solutions with initial values and control items. In Section 3, we discuss the optimal control of the viscous weakly dispersive BBM equation and prove the existence of the optimal control.

\section{Existence and uniqueness of weak solution for the viscous weakly dispersive BBM equation}

For fixed $T>0$, set $\Omega=(0,1), Q=(0, T) \times \Omega$ and $Q_{0} \subseteq Q$ be an open set with positive measure. Let $V=H_{0}^{1}(0,1), H=L^{2}(0,1), V^{*}=H^{-1}(0,1)$ and $H^{*}=L^{2}(0,1)$ are dual space respectively. It is well known that $V$ is dense in $H$, and

$$
V \hookrightarrow H=H^{*} \hookrightarrow V^{*}
$$

moreover, the embedding being dense. The extended operator $B^{*} \in \mathcal{L}\left(L^{2}\left(Q_{0}\right)\right.$, $\left.L^{2}\left(V^{*}\right)\right)$ is introduced as

$$
B^{*} q= \begin{cases}q, & q \in Q_{0}, \\ 0, & q \in Q \backslash Q_{0} .\end{cases}
$$

We also supply $H$ with the inner product $(\cdot, \cdot)_{H}$ and the norm $\|\cdot\|_{H}$, and define $\|u\|_{H^{m}(\Omega)}=\left\|D^{m} u\right\|_{H}$, here $D^{m}=\partial^{m} / \partial x^{m}(m=0,1,2, \ldots)$. A new space $W(0, T ; V)$ is introduced as

$$
W(0, T ; V)=\left\{f: f \in L^{2}(0, T ; V), f_{t} \in L^{2}\left(0, T ; V^{*}\right)\right\},
$$

which is a Hilbert space endowed with common inner product.

Now, consider the following viscous weakly dispersive BBM equation

$$
\left\{\begin{array}{l}
u_{t}-u_{x x t}-\varepsilon\left(u-u_{x x}\right)_{x x}+u_{x}+u u_{x}+\lambda\left(u-u_{x x}\right)_{x}=f+B^{*} \bar{\omega}, \\
u(x, 0)=u_{0}(x), \\
u(0, t)=u(1, t)=u_{x}(0, t)=u_{x}(1, t)=u_{x x}(0, t)=u_{x x}(1, t)=0,
\end{array}\right.
$$

where $x \in(0,1), t \in[0, T], f+B^{*} \bar{\omega} \in L^{2}\left(0, T ; V^{*}\right)$. Setting $y=u-u_{x x}$, from (2.1), we obtain a quasi-linear evolution equation:

$$
\left\{\begin{array}{l}
y_{t}-\varepsilon y_{x x}+\lambda y_{x}+u_{x} y+u_{x}-u_{x} u_{x x}=f+B^{*} \bar{\omega} \\
y(x, 0)=u(x, 0)-u_{x x}(x, 0)=\psi, \\
u(0, t)=u(1, t)=u_{x}(0, t)=u_{x}(1, t)=u_{x x}(0, t)=u_{x x}(1, t)=0 .
\end{array}\right.
$$

In order to prove the existence of weak solution to the viscous weakly dispersive BBM equation, we give the definition of the weak solution in the space $W(0, T ; V)$. 
Definition 2.1. A function $y(x, t) \in W(0, T ; V)$ is called a weak solution to Eqs.(2.2), if

$$
\begin{aligned}
& \frac{d}{d t}(y, v)_{H}+\varepsilon\left(y_{x}, v_{x}\right)_{H}+\lambda\left(y_{x}, v\right)_{H}+\left(u_{x} y, v\right)_{H}+\left(u_{x}, v\right)_{H}-\left(u_{x} u_{x x}, v\right)_{H} \\
= & \left\langle f+B^{*} \bar{\omega}, v\right\rangle_{V^{*}, V}
\end{aligned}
$$

for all $v \in V$, a.e. $t \in[0, T]$ and $y(x, 0)=\psi$.

Remark 2.1. Since $W(0, T ; V)$ is compactly embedded into $C(0, T ; H)$ ([6], $[19])$, then $y(x, 0)=\psi \in H$ is valid.

Theorem 2.1. Let $\psi \in H, f \in L^{2}\left(0, T ; V^{*}\right)$. Then for any $\bar{\omega} \in L^{2}\left(Q_{0}\right)$, there exists a unique weak solution to the Eqs.(2.2) in the interval $[0, T]$.

Proof. Since $-\partial_{x}^{2}$ is a Laplacian operator with one dimension, we can choose the base functions $\left\{w_{j}\right\}_{j \in N}$ in $V$ with $w_{j}$ being the eigenfunctions subject to the Dirichlet condition:

$$
\left\{\begin{array}{l}
-\partial_{x}^{2} w_{j}=\lambda_{j} w_{j}, \quad \text { in } \Omega \\
w_{j}=0, \quad \text { on } \partial \Omega .
\end{array}\right.
$$

We also normalize $w_{j}$ such that $\left\|w_{j}\right\|_{H}=1[19]$. By the elliptic operator theory, $\left\{w_{j}\right\}$ forms base functions in $V$. Now we use the Faedo-Galerkin method to find the approximate solution.

For $\forall m \in N$, define the ansatz space by

$$
V_{m}=\operatorname{span}\left\{w_{1}, w_{2}, \ldots, w_{m}\right\} \subseteq V .
$$

Set

$$
y_{m}=\sum_{i=1}^{m} y_{i}^{m}(t) w_{i}(x),
$$

which satisfied the following identities:

(2.5)

$\left\{\begin{array}{l}y_{m t}-\varepsilon y_{m x x}+\lambda y_{m x}+u_{m x} y_{m}+u_{m x}-u_{m x} u_{m x x}=f+B^{*} \bar{\omega}, \\ y_{m}(x, 0)=\psi_{m}, \\ u_{m}(0, t)=u_{m}(1, t)=u_{m x}(0, t)=u_{m x}(1, t)=u_{m x x}(0, t)=u_{m x x}(1, t)=0,\end{array}\right.$

where $y_{m}=u_{m}-u_{m x x}$ and $y_{m}(0)=\psi_{m} \rightarrow \psi$ strongly in $H$ as $m \rightarrow \infty$.

Now, we prove the existence of weak solution by analyzing the limiting behavior of sequences of smooth functions $\left\{u_{m}\right\}$ and $\left\{y_{m}\right\}$.

Since Eqs.(2.5) are first-order differential equations, thus by the usual Picard iteration method used in ODE, one can conclude that there exists a $t_{m}>0$ such that, the problem Eqs.(2.5) admits a unique local solution in $\left[0, t_{m}\right]$. We will show that the solution is uniformly bounded as $t_{m} \rightarrow T$. 
THE VISCOUS WEAKLY DISPERSIVE BENJAMIN-BONA-MAHONY EQUATION 1189

Multiplied Eqs.(2.5) both sides by $u_{m}$ and integrating with respect to $x$ over $\Omega$, we get

(2.6) $\frac{1}{2} \frac{d}{d t}\left(\left\|u_{m}\right\|_{H}^{2}+\left\|u_{m}\right\|_{V}^{2}\right)+\varepsilon\left(\left\|u_{m}\right\|_{V}^{2}+\left\|u_{m}\right\|_{H^{2}}^{2}\right)=\left\langle f+B^{*} \bar{\omega}, u_{m}\right\rangle_{V^{*}, V}$.

Integrating (2.6) from 0 to $t$, we have

$$
\begin{aligned}
& \frac{1}{2}\left(\left\|u_{m}\right\|_{H}^{2}+\left\|u_{m}\right\|_{V}^{2}\right)+\varepsilon \int_{0}^{t}\left(\left\|u_{m}\right\|_{V}^{2}+\left\|u_{m}\right\|_{H^{2}}^{2}\right) d s \\
= & \int_{0}^{t}\left\langle f+B^{*} \bar{\omega}, u_{m}\right\rangle_{V^{*}, V} d s+\frac{1}{2}\left(\left\|u_{m}(0)\right\|_{H}^{2}+\left\|u_{m}(0)\right\|_{V}^{2}\right) .
\end{aligned}
$$

Since $f+B^{*} \bar{\omega} \in L^{2}\left(0, T ; V^{*}\right)$, so there exist a constant $C_{1} \geq 0$ such that

$$
\left\|f+B^{*} \bar{\omega}\right\|_{L^{2}\left(0, T ; V^{*}\right)} \leq C_{1},
$$

and $M_{1} \geq 0$ such that

$$
\frac{1}{2}\left(\left\|u_{m}(0)\right\|_{H}^{2}+\left\|u_{m}(0)\right\|_{V}^{2}\right) \leq M_{1} .
$$

By Young's inequality, we obtain

$$
\begin{aligned}
\left|\int_{0}^{t}\left\langle f+B^{*} \bar{\omega}, u_{m}\right\rangle_{V^{*}, V} d s\right| & \leq \int_{0}^{t}\left\|f+B^{*} \bar{\omega}\right\|_{V^{*}}\left\|u_{m}\right\|_{V} d s \\
& \leq \frac{1}{\varepsilon} \int_{0}^{t}\left\|f+B^{*} \bar{\omega}\right\|_{V^{*}}^{2} d s+\varepsilon \int_{0}^{t}\left\|u_{m}\right\|_{V}^{2} d s \\
& \leq \frac{1}{\varepsilon}\left\|f+B^{*} \bar{\omega}\right\|_{L^{2}\left(0, T ; V^{*}\right)}^{2}+\varepsilon \int_{0}^{t}\left\|u_{m}\right\|_{V^{2}}^{2} d s \\
& \leq \frac{C_{1}^{2}}{\varepsilon}+\varepsilon \int_{0}^{t}\left\|u_{m}\right\|_{V}^{2} d s .
\end{aligned}
$$

It follows from (2.7)-(2.9) that

$\frac{1}{2}\left(\left\|u_{m}\right\|_{H}^{2}+\left\|u_{m}\right\|_{V}^{2}\right)+\varepsilon \int_{0}^{t}\left(\left\|u_{m}\right\|_{V}^{2}+\left\|u_{m}\right\|_{H^{2}}^{2}\right) d s \leq \varepsilon \int_{0}^{t}\left\|u_{m}\right\|_{V}^{2} d s+\frac{C_{1}^{2}}{\varepsilon}+M_{1}$.

So,

$$
\left\|u_{m}\right\|_{H}^{2}+\left\|u_{m}\right\|_{V}^{2} \leq \frac{2}{\varepsilon} C_{1}^{2}+2 M_{1}=C_{2}^{2}
$$

for $\forall t \in[0, T]$, and $C_{2} \geq 0$ is a constant. Which implies, by (2.11),

$$
\left\|u_{m}\right\|_{H} \leq C_{2}, \quad\left\|u_{m}\right\|_{V} \leq C_{2} .
$$

Again, multiplied Eqs.(2.5) both sides by $-u_{m x x}$ and integrating on $(0, t) \times \Omega$, we obtain

$$
\begin{aligned}
& \frac{1}{2}\left(\left\|u_{m}\right\|_{V}^{2}+\left\|u_{m}\right\|_{H^{2}}^{2}\right)+\varepsilon \int_{0}^{t}\left(\left\|u_{m}\right\|_{H^{2}}^{2}+\left\|u_{m}\right\|_{H^{3}}^{2}\right) d s \\
= & -\int_{0}^{t}\left\langle f+B^{*} \bar{\omega}, u_{m x x}\right\rangle_{V^{*}, V} d s+\int_{0}^{t}\left(u_{m} u_{m x}, u_{m x x}\right)_{H} d s
\end{aligned}
$$




$$
+\frac{1}{2}\left(\left\|u_{m}(0)\right\|_{V}^{2}+\left\|u_{m}(0)\right\|_{H^{2}}^{2}\right) .
$$

Thus, from (2.8), we have

$$
\begin{aligned}
& \left|\int_{0}^{t}\left\langle f+B^{*} \bar{\omega}, u_{m x x}\right\rangle_{V^{*}, V} d s\right| \\
\leq & \int_{0}^{t}\left\|f+B^{*} \bar{\omega}\right\|_{V^{*}}\left\|u_{m}\right\|_{H^{3}} d s \\
\leq & \frac{1}{\varepsilon}\left\|f+B^{*} \bar{\omega}\right\|_{L^{2}\left(0, T ; V^{*}\right)}^{2}+\varepsilon \int_{0}^{t}\left\|u_{m}\right\|_{H^{3}}^{2} d s \\
\leq & \frac{C_{1}^{2}}{\varepsilon}+\varepsilon \int_{0}^{t}\left\|u_{m}\right\|_{H^{3}}^{2} d s .
\end{aligned}
$$

By Poincaré inequality and Sobolev embedding theorem, we get

$$
\begin{aligned}
\left|\int_{0}^{t}\left(u_{m} u_{m x}, u_{m x x}\right)_{H} d s\right| & \leq \int_{0}^{t}\left\|u_{m}\right\|\left\|_{H}\right\| u_{m x}\left\|_{L^{\infty}}\right\| u_{m x x} \|_{H} d s \\
& \leq k C_{2} \int_{0}^{t}\left\|u_{m x}\right\|_{V}\left\|u_{m}\right\|_{H^{2}} d s \\
& \leq k C_{2} \int_{0}^{t}\left\|u_{m}\right\|_{H^{2}}^{2} d s,
\end{aligned}
$$

where $k$ is an embedding constant. From (2.12)-(2.14), we obtain

$$
\left\|u_{m}\right\|_{V}^{2}+\left\|u_{m}\right\|_{H^{2}}^{2} \leq 2 k C_{2} \int_{0}^{t}\left\|u_{m}\right\|_{H^{2}}^{2} d s+\frac{2 C_{1}^{2}}{\varepsilon}+2 M_{2},
$$

where $M_{2}$ satisfies

$$
\frac{1}{2}\left(\left\|u_{m}(0)\right\|_{V}^{2}+\left\|u_{m}(0)\right\|_{H^{2}}^{2}\right) \leq M_{2} .
$$

By using Gronwall's inequality, we get from (2.15) that

$$
\left\|u_{m}\right\|_{H^{2}}^{2} \leq\left(\frac{2 C_{1}^{2}}{\varepsilon}+2 M_{2}\right) e^{2 k T C_{2}}=C_{3}^{2} .
$$

Thus we know that $\left\|u_{m}\right\|_{H^{2}} \leq C_{3}$.

Now, we will give a uniform $L^{2}(0, T ; V)$ bound on the sequence $\left\{y_{m}\right\}$.

Multiplied Eqs.(2.5) both sides by $y_{m}$ and integrating over $\Omega$, yields that

$$
\begin{aligned}
& \frac{1}{2} \frac{d}{d t}\left\|y_{m}\right\|_{H}^{2}+\varepsilon\left\|y_{m}\right\|_{V}^{2}+\int_{0}^{1} u_{m x} y_{m}^{2} d x-\int_{0}^{1} u_{m x} u_{m x x} y_{m} d x \\
= & \left\langle f+B^{*} \bar{\omega}, y_{m}\right\rangle_{V^{*}, V} .
\end{aligned}
$$

By Poincaré inequality and Sobolev embedding theorem, we have

$$
\begin{aligned}
\left|\int_{0}^{1} u_{m x} y_{m}^{2} d x\right| & =2\left|\int_{0}^{1} u_{m} y_{m x} y_{m} d x\right| \\
& \leq 2\left\|u_{m}\right\|_{L^{\infty}}\left\|y_{m}\right\|_{H}\left\|y_{m}\right\|_{V}
\end{aligned}
$$


THE VISCOUS WEAKLY DISPERSIVE BENJAMIN-BONA-MAHONY EQUATION 1191

$$
\begin{aligned}
& \leq 2 k\left\|u_{m}\right\|_{V}\left\|y_{m}\right\|_{H}\left\|y_{m}\right\|_{V} \\
& \leq 2 k C_{2}\left\|y_{m}\right\|_{H}\left\|y_{m}\right\|_{V},
\end{aligned}
$$

and

$$
\begin{aligned}
\left|\int_{0}^{1} u_{m x} u_{m x x} y_{m} d x\right| & =\frac{1}{2}\left|\int_{0}^{1} u_{m x}^{2} y_{m x} d x\right| \\
& \leq \frac{1}{2}\left\|u_{m x}\right\|_{L^{\infty}}\left\|u_{m x}\right\|_{H}\left\|y_{m x}\right\|_{H} \\
& \leq \frac{k}{2}\left\|u_{m x}\right\|_{V}\left\|u_{m}\right\|_{V}\left\|y_{m}\right\|_{V} \\
& \leq \frac{k C_{2} C_{3}}{2}\left\|y_{m}\right\|_{V} .
\end{aligned}
$$

It then follows from (2.17)-(2.19) and Young's inequality that

$$
\begin{aligned}
\frac{d}{d t}\left\|y_{m}\right\|_{H}^{2}+2 \varepsilon\left\|y_{m}\right\|_{V}^{2} \leq & \left(2\left\|f+B^{*} \bar{\omega}\right\|_{V^{*}}+k C_{2} C_{3}\right)\left\|y_{m}\right\|_{V}+4 k C_{2}\left\|y_{m}\right\|_{H}\left\|y_{m}\right\|_{V} \\
\leq & \frac{2}{\varepsilon}\left(2\left\|f+B^{*} \bar{\omega}\right\|_{V^{*}}+k C_{2} C_{3}\right)^{2}+\frac{\varepsilon}{2}\left\|y_{m}\right\|_{V}^{2} \\
& +\frac{32}{\varepsilon} k^{2} C_{2}^{2}\left\|y_{m}\right\|_{H}^{2}+\frac{\varepsilon}{2}\left\|y_{m}\right\|_{V}^{2} .
\end{aligned}
$$

In view of $(a+b)^{2} \leq 2\left(a^{2}+b^{2}\right)$, from above inequality, we have (2.20) $\frac{d}{d t}\left\|y_{m}\right\|_{H}^{2}+\varepsilon\left\|y_{m}\right\|_{V}^{2} \leq \frac{4}{\varepsilon}\left(4\left\|f+B^{*} \bar{\omega}\right\|_{V^{*}}^{2}+k^{2} C_{2}^{2} C_{3}^{2}\right)+\frac{32}{\varepsilon} k^{2} C_{2}^{2}\left\|y_{m}\right\|_{H}^{2}$. Integrating $(2.20)$ on interval $[0, t]$, we obtain

$$
\left\|y_{m}\right\|_{H}^{2}+\varepsilon \int_{0}^{t}\left\|y_{m}\right\|_{V}^{2} d s \leq M_{3}+M_{4} \int_{0}^{t}\left\|y_{m}\right\|_{H}^{2} d s
$$

where $M_{3}=\frac{4}{\varepsilon}\left(4 C_{1}^{2}+k^{2} T C_{2}^{2} C_{3}^{2}\right)+\|\psi\|_{H}^{2}, M_{4}=\frac{32}{\varepsilon} k^{2} C_{2}^{2}$ are positive constants.

By using Gronwall's inequality to (2.21), there exists a $M_{5}>0$ such that

$$
\left\|y_{m}\right\|_{H} \leq M_{5} \text {. }
$$

Thus, combine (2.21) with (2.22), there exists a $M_{6}>0$ such that

$$
\left\|y_{m}\right\|_{L^{2}(0, T ; V)} \leq M_{6} \text {. }
$$

In what follows, we shall give a uniform $L^{2}\left(0, T ; V^{*}\right)$ bound on the sequence $\left\{y_{m t}\right\}$.

By Eqs.(2.5) and Sobolev embedding theorem, we obtain

$$
\begin{aligned}
\left\|y_{m t}\right\|_{V^{*}}= & \sup _{\|\phi\|_{V}=1}\left\langle y_{m t}, \phi\right\rangle_{V^{*}, V} \\
\leq & \left\|f+B^{*} \bar{\omega}\right\|_{V^{*}}+\varepsilon\left\|y_{m}\right\|_{V}+\lambda\left\|y_{m}\right\|_{H}+\left\|u_{m}\right\|_{H}\left\|y_{m}\right\|_{V}+\left\|u_{m}\right\|_{H} \\
& +\frac{k}{2}\left\|u_{m}\right\|_{H^{2}}^{2}
\end{aligned}
$$

$$
\leq\left\|f+B^{*} \bar{\omega}\right\|_{V^{*}}+\left(\varepsilon+C_{2}\right)\left\|y_{m}\right\|_{V}+C_{2}+\frac{k C_{3}^{2}}{2}+\lambda M_{5} .
$$


It follows from (2.24) that

(2.25) $\left\|y_{m t}\right\|_{V^{*}}^{2} \leq 3\left\|f+B^{*} \bar{\omega}\right\|_{V^{*}}^{2}+3\left(\varepsilon+C_{2}\right)^{2}\left\|y_{m}\right\|_{V}^{2}+3\left(C_{2}+\frac{k}{2} C_{3}^{2}+\lambda M_{5}\right)^{2}$.

Integrating the above inequality with respect to $t$ on $[0, T]$, we get

$$
\begin{array}{rl}
\left\|y_{m t}\right\|_{L^{2}\left(0, T ; V^{*}\right) \leq}^{2} & 3\left\|f+B^{*} \bar{\omega}\right\|_{L^{2}\left(0, T ; V^{*}\right)}^{2}+3\left(\varepsilon+C_{2}\right)^{2}\left\|y_{m}\right\|_{L^{2}(0, T ; V)}^{2} \\
& +3 T\left(C_{2}+\frac{k}{2} C_{3}^{2}+\lambda M_{5}\right)^{2} \\
\leq & 3 C_{1}^{2}+3 M_{6}^{2}\left(\varepsilon+C_{2}\right)^{2}+3 T\left(C_{2}+\frac{k}{2} C_{3}^{2}+\lambda M_{5}\right)^{2} \\
= & M_{7}^{2} .
\end{array}
$$

Thus, from (2.23) and (2.26), we have

(2.27) $\left\|y_{m}\right\|_{W(0, T ; V)}^{2}=\left\|y_{m}\right\|_{L^{2}(0, T ; V)}^{2}+\left\|y_{m t}\right\|_{L^{2}\left(0, T ; V^{*}\right)}^{2} \leq M_{6}^{2}+M_{7}^{2}<\infty$.

So, there exists a subsequence of $\left\{y_{m}\right\}$, without loss of generality, denote the subsequence by $\left\{y_{m}\right\}$, such that

$$
y_{m} \rightarrow y \quad \text { weakly in } L^{2}(0, T ; V),
$$

and

$$
\frac{d}{d t} y_{m} \rightarrow \frac{d}{d t} y \quad \text { weakly in } L^{2}\left(0, T ; V^{*}\right)
$$

Since $W(0, T ; V)$ is compactly embedded into $L^{2}\left(0, T ; L^{\infty}(\Omega)\right)[14]$, we have

$$
y_{m} \rightarrow y \quad \text { strongly in } L^{2}\left(0, T ; L^{\infty}(\Omega)\right) \text {. }
$$

By Remark 2.1, it is shown that

$$
y_{m} \rightarrow y \quad \text { strongly in } C(0, T ; H) \text {. }
$$

Moreover,

$$
u_{m} \rightarrow u, \quad u_{m x} \rightarrow u_{x}, \quad u_{m x x} \rightarrow u_{x x}, \quad \text { strongly in the space } C(0, T ; H) .
$$

In following, we are going to verify the $y$ is a solution of (2.2). Setting $\eta=\xi(t) \nu \in \mathscr{D}(0, T ; V)$, here $\xi(t) \in \mathscr{D}(0, T), \nu \in V$. From $(2.5)$, we obtain

$$
\begin{aligned}
& \int_{0}^{T}\left\langle f+B^{*} \bar{\omega}, \eta\right\rangle_{V^{*}, V} d t \\
= & \int_{0}^{T}\left\langle y_{m t}, \eta\right\rangle_{V^{*}, V} d t+\varepsilon \int_{0}^{T}\left(y_{m x}, \eta_{x}\right)_{H} d t+\lambda \int_{0}^{T}\left(y_{m x}, \eta\right)_{H} d t \\
& +\int_{0}^{T}\left(u_{m x} y_{m}, \eta\right)_{H} d t+\int_{0}^{T}\left(u_{m x}, \eta\right)_{H} d t-\int_{0}^{T}\left(u_{m x} u_{m x x}, \eta\right)_{H} d t .
\end{aligned}
$$

Again, from (2.28)-(2.31), we have

$$
\left|\int_{0}^{T}\left(u_{m x} y_{m}-u_{x} y, \varphi\right)_{H} d t\right|
$$


THE VISCOUS WEAKLY DISPERSIVE BENJAMIN-BONA-MAHONY EQUATION 1193

$$
\begin{aligned}
\leq & \left|\int_{0}^{T}\left(u_{m x}\left(y_{m}-y\right), \varphi\right)_{H} d t\right|+\left|\int_{0}^{T}\left(\left(u_{m x}-u_{x}\right) y, \varphi\right)_{H} d t\right| \\
\leq & \int_{0}^{T}\left\|u_{m}\right\|_{H}\|\varphi\|_{V}\left\|y_{m}-y\right\|_{L^{\infty}} d t+\int_{0}^{T}\left\|u_{m}-u\right\|_{H}\|\varphi\|_{V}\|y\|_{L^{\infty}} d t \\
\leq & \left\|u_{m}\right\|_{C(0, T ; H)}\|\varphi\|_{L^{2}(0, T ; V)}\left\|y_{m}-y\right\|_{L^{2}\left(0, T ; L^{\infty}\right)} \\
& \quad+\|y\|_{L^{2}\left(0, T ; L^{\infty}\right)}\|\varphi\|_{L^{2}(0, T ; V)}\left\|u_{m}-u\right\|_{C(0, T ; H)} \\
\longrightarrow & 0, \quad m \rightarrow+\infty, \forall \varphi \in L^{2}(0, T ; V)
\end{aligned}
$$

and

$$
\begin{aligned}
\left|\int_{0}^{T}\left(u_{m x} u_{m x x}-u_{x} u_{x x}, \varphi\right)_{H} d t\right| & =\frac{1}{2}\left|\int_{0}^{T}\left(\left(u_{m x}\right)^{2}-\left(u_{x}\right)^{2}, \varphi\right)_{H} d t\right| \\
& \leq \frac{1}{2}\left|\int_{0}^{T}\left\|\left(u_{m x}\right)^{2}-\left(u_{x}\right)^{2}\right\|_{H}\|\varphi\|_{H} d t\right| \\
& \leq \frac{1}{2} T^{\frac{1}{2}}\left\|\left(u_{m x}\right)^{2}-\left(u_{x}\right)^{2}\right\|_{C(0, T ; H)}\|\varphi\|_{L^{2}(0, T ; H)} \\
& \longrightarrow 0, \quad m \rightarrow+\infty, \forall \varphi \in L^{2}(0, T ; V) .
\end{aligned}
$$

Letting $m \rightarrow+\infty$ in (2.32), yields that

$$
\begin{aligned}
& \int_{0}^{T}\left\langle f+B^{*} \bar{\omega}, \eta\right\rangle_{V^{*}, V} d t \\
= & \int_{0}^{T}\left\langle y_{t}, \eta\right\rangle_{V^{*}, V} d t+\varepsilon \int_{0}^{T}\left(y_{x}, \eta_{x}\right)_{H} d t+\lambda \int_{0}^{T}\left(y_{x}, \eta\right)_{H} d t \\
& +\int_{0}^{T}\left(u_{x} y, \eta\right)_{H} d t+\int_{0}^{T}\left(u_{x}, \eta\right)_{H} d t-\int_{0}^{T}\left(u_{x} u_{x x}, \eta\right)_{H} d t .
\end{aligned}
$$

Since $\mathscr{D}(0, T ; V)$ is dense in $L^{2}(0, T ; V)$, the above equality holds for all functions $\eta \in L^{2}(0, T ; V)$. Hence, we have specially

$$
\begin{aligned}
& \frac{d}{d t}(y, \eta)_{H}+\varepsilon\left(y_{x}, \eta_{x}\right)_{H}+\lambda\left(y_{x}, \eta\right)_{H}+\left(u_{x} y, \eta\right)_{H}+\left(u_{x}, \eta\right)_{H}-\left(u_{x} u_{x x}, \eta\right)_{H} \\
= & \left\langle f+B^{*} \bar{\omega}, \eta\right\rangle_{V^{*}, V}
\end{aligned}
$$

for each $\eta \in V$ and a.e. $t \in[0, T]$.

From $(2.31)$, we have $y_{m}(0) \rightarrow y(0)$ strongly in $H$. So, it also weakly convergence in $H$, by the uniqueness of the limit, we have

$$
y(x, 0)=\psi .
$$

On the other hand, it is clear that the uniqueness of the weak solution follows from the inequality (2.20) immediately.

Thus the proof is therefore complete.

In the following, we shall establish the inequality for the norm of weak solution with initial value and control item, which is necessary in discussing the exitance of optimal control. 
Theorem 2.2. Let $\psi \in H, f \in L^{2}\left(0, T ; V^{*}\right)$. Then for $\forall \bar{\omega} \in L^{2}\left(Q_{0}\right)$, there exists constants $L_{1}, L_{2} \geq 0$, such that

$$
\|y\|_{W(0, T ; V)}^{2} \leq L_{1}\left(\left\|f+B^{*} \bar{\omega}\right\|_{L^{2}\left(0, T ; V^{*}\right)}^{2}+\|\psi\|_{H}^{2}\right)+L_{2} .
$$

Proof. Multiplied Eqs.(2.2) by $u$, and integrating over $\Omega$, which implies

$$
\frac{1}{2} \frac{d}{d t}\left(\|u\|_{H}^{2}+\|u\|_{V}^{2}\right)+\varepsilon\left(\|u\|_{V}^{2}+\|u\|_{H^{2}}^{2}\right)=\left\langle f+B^{*} \bar{\omega}, u\right\rangle_{V^{*}, V} .
$$

Similar to the argument in the Theorem 2.1, we have

$$
\|u\|_{H} \leq C_{2}, \quad\|u\|_{V} \leq C_{2},
$$

where $C_{2}$ is a constant the same as in Theorem 2.1.

Similarly, multiplied Eqs.(2.2) by $-u_{x x}$, and integrating over $\Omega$, we obtain

$$
\left\|u_{m}\right\|_{H^{2}} \leq C_{3},
$$

where $C_{3}$ is also a constant from (2.16).

Again, multiplied Eqs.(2.2) by $y$ and integrating over $\Omega$, which yields

$$
\frac{1}{2} \frac{d}{d t}\|y\|_{H}^{2}+\varepsilon\|y\|_{V}^{2}+\int_{0}^{1} u_{x} y^{2} d x-\int_{0}^{1} u_{x} u_{x x} y d x=\left\langle f+B^{*} \bar{\omega}, y\right\rangle_{V^{*}, V}
$$

since

$$
\left|\int_{0}^{1} u_{x} y^{2} d x\right| \leq 2 k\|u\|_{V}\|y\|_{H}\|y\|_{V} \leq 2 k C_{2}\|y\|_{H}\|y\|_{V}
$$

$\left|\int_{0}^{1} u_{x} u_{x x} y d x\right| \leq \frac{1}{2}\left\|u_{x}\right\|_{L^{\infty}}\left\|u_{x}\right\|_{H}\left\|y_{x}\right\|_{H} \leq \frac{k}{2}\|u\|_{H^{2}}\|u\|_{V}\|y\|_{V} \leq \frac{k C_{2} C_{3}}{2}\|y\|_{V}$.

From (2.38)-(2.40), we get

(2.41) $\frac{1}{2} \frac{d}{d t}\|y\|_{H}^{2}+\varepsilon\|y\|_{V}^{2} \leq\left(\left\|f+B^{*} \bar{\omega}\right\|_{V^{*}}+\frac{k C_{2} C_{3}}{2}\right)\|y\|_{V}+2 k C_{2}\|y\|_{H}\|y\|_{V}$.

Using Young's inequality yields

$$
\begin{aligned}
\frac{1}{2} \frac{d}{d t}\|y\|_{H}^{2}+\varepsilon\|y\|_{V}^{2} & \leq\left(\left\|f+B^{*} \bar{\omega}\right\|_{V^{*}}+\frac{k C_{2} C_{3}}{2}\right)\|y\|_{V}+2 k C_{2}\|y\|_{H}\|y\|_{V} \\
& \leq \frac{2}{\varepsilon}\left(\left\|f+B^{*} \bar{\omega}\right\|_{V^{*}}+\frac{k C_{2} C_{3}}{2}\right)^{2}+\frac{8}{\varepsilon} k^{2} C_{2}^{2}\|y\|_{H}^{2}+\varepsilon\|y\|_{V}^{2},
\end{aligned}
$$

then

$$
\frac{1}{2} \frac{d}{d t}\|y\|_{H}^{2} \leq \frac{2}{\varepsilon}\left(\left\|f+B^{*} \bar{\omega}\right\|_{V^{*}}+\frac{k C_{2} C_{3}}{2}\right)^{2}+\frac{8}{\varepsilon} k^{2} C_{2}^{2}\|y\|_{H}^{2} .
$$

Using Gronwall' inequality to the last inequality, we have

$$
\begin{aligned}
\|y\|_{H}^{2} & \leq \frac{\varepsilon e^{\frac{16}{\varepsilon} k^{2} C_{2}^{2} T}}{8 k^{2} C_{2}^{2}}\left[\frac{2}{\varepsilon}\left(\left\|f+B^{*} \bar{\omega}\right\|_{V^{*}}+\frac{k C_{2} C_{3}}{2}\right)^{2}+\frac{8}{\varepsilon} k^{2} C_{2}^{2}\|\psi\|_{H}^{2}\right] \\
& \leq \frac{\varepsilon e^{\frac{16}{\varepsilon} k^{2} C_{2}^{2} T}}{8 k^{2} C_{2}^{2}}\left[\frac{4}{\varepsilon}\left(\left\|f+B^{*} \bar{\omega}\right\|_{V^{*}}^{2}+\frac{k^{2} C_{2}^{2} C_{3}^{2}}{4}\right)+\frac{8}{\varepsilon} k^{2} C_{2}^{2}\|\psi\|_{H}^{2}\right]
\end{aligned}
$$


THE VISCOUS WEAKLY DISPERSIVE BENJAMIN-BONA-MAHONY EQUATION 1195

$$
\leq C_{4}\left(\left\|f+B^{*} \bar{\omega}\right\|_{V^{*}}^{2}+\|\psi\|_{H}^{2}\right)+C_{5}
$$

where

$$
C_{4}=\max \left\{\frac{e^{\frac{16}{\varepsilon} k^{2} C_{2}^{2} T}}{2 k^{2} C_{2}^{2}}, e^{\frac{16}{\varepsilon} k^{2} C_{2}^{2} T}\right\}, \quad C_{5}=\frac{\varepsilon e^{\frac{16}{\varepsilon} k^{2} C_{2}^{2} T} C_{3}^{2}}{32} .
$$

In addition, by using Young's inequality, (2.41) and (2.42), which yields

$$
\begin{aligned}
& \frac{d}{d t}\|y\|_{H}^{2}+\varepsilon\|y\|_{V}^{2} \\
\leq & \frac{8}{\varepsilon}\left[\left(\left\|f+B^{*} \bar{\omega}\right\|_{V^{*}}+\frac{k C_{2} C_{3}}{2}\right)^{2}+4 k^{2} C_{2}^{2}\|y\|_{H}^{2}\right] \\
\leq & \frac{8}{\varepsilon}\left(2\left\|f+B^{*} \bar{\omega}\right\|_{V^{*}}^{2}+\frac{k^{2} C_{2}^{2} C_{3}^{2}}{2}+4 k^{2} C_{2}^{2}\|y\|_{H}^{2}\right) \\
\leq & \left(\frac{16}{\varepsilon}+\frac{32 k^{2} C_{2}^{2} C_{4}}{\varepsilon}\right)\left\|f+B^{*} \bar{\omega}\right\|_{V^{*}}^{2}+\frac{32 k^{2} C_{2}^{2} C_{4}}{\varepsilon}\|\psi\|_{H}^{2} \\
& +\frac{32 k^{2} C_{2}^{2} C_{5}}{\varepsilon}+\frac{4 k^{2} C_{2}^{2} C_{3}^{2}}{\varepsilon} \\
\leq & C_{6}\left(\left\|f+B^{*} \bar{\omega}\right\|_{V^{*}}^{2}+\|\psi\|_{H}^{2}\right)+C_{7},
\end{aligned}
$$

where $C_{6}=\max \left\{\frac{16}{\varepsilon}+\frac{32 k^{2} C_{2}^{2} C_{4}}{\varepsilon}, \frac{32 k^{2} C_{2}^{2} C_{4}}{\varepsilon}\right\}, C_{7}=\frac{32 k^{2} C_{2}^{2} C_{5}}{\varepsilon}+\frac{4 k^{2} C_{2}^{2} C_{3}^{2}}{\varepsilon}$.

Hence, integrating $(2.43)$ over $[0, t]$, we have

$$
\begin{aligned}
\|y\|_{L^{2}(0, T ; V)}^{2} & \leq \frac{C_{6}}{\varepsilon}\left\|f+B^{*} \bar{\omega}\right\|_{L^{2}\left(0, T ; V^{*}\right)}^{2}+\frac{1+C_{6} T}{\varepsilon}\|\psi\|_{H}^{2}+\frac{C_{7} T}{\varepsilon} \\
& \leq C_{8}\left(\left\|f+B^{*} \bar{\omega}\right\|_{L^{2}\left(0, T ; V^{*}\right)}^{2}+\|\psi\|_{H}^{2}\right)+C_{9},
\end{aligned}
$$

where $C_{8}=\max \left\{\frac{C_{6}}{\varepsilon}, \frac{1+C_{6} T}{\varepsilon}\right\}$, and $C_{9}=\frac{C_{7} T}{\varepsilon}$.

Meantime, from Eqs.(2.2), we get

$$
\begin{aligned}
\left\|y_{t}\right\|_{V^{*}}= & \sup _{\|\phi\|_{V}=1}\left\langle y_{t}, \phi\right\rangle_{V^{*}, V} \\
\leq & \left\|f+B^{*} \bar{\omega}\right\|_{V^{*}}+\varepsilon\|y\|_{V}+\lambda\|y\|_{H}+\|u\|_{H}\|y\|_{V}+\|u\|_{H} \\
& \quad+\frac{k}{2}\|u\|_{H^{2}}^{2} \\
\leq & \left\|f+B^{*} \bar{\omega}\right\|_{V^{*}}+\left(\varepsilon+C_{2}\right)\|y\|_{V}+\lambda\|y\|_{H}+C_{2}+\frac{k}{2} C_{3}^{2} .
\end{aligned}
$$

By using Schwarz inequality and (2.37), we obtain

$$
\begin{aligned}
\left\|y_{t}\right\|_{V^{*}}^{2} \leq & \left(\left\|f+B^{*} \bar{\omega}\right\|_{V^{*}}+\left(\varepsilon+C_{2}\right)\|y\|_{V}+\lambda\|y\|_{H}+C_{2}+\frac{k}{2} C_{3}^{2}\right)^{2} \\
\leq & 4\left\|f+B^{*} \bar{\omega}\right\|_{V^{*}}^{2}+4\left(\varepsilon+C_{2}\right)^{2}\|y\|_{V}^{2}+4 \lambda^{2}\|y\|_{H}^{2}+4\left(C_{2}+\frac{k}{2} C_{3}^{2}\right)^{2} \\
\leq & \left(4+4 \lambda^{2} C_{4}\right)\left(\left\|f+B^{*} \bar{\omega}\right\|_{V^{*}}^{2}+\|\psi\|_{H}^{2}\right)+4\left(\varepsilon+C_{2}\right)^{2}\|y\|_{V}^{2} \\
& +4\left(C_{2}+\frac{k}{2} C_{3}^{2}\right)^{2}+4 \lambda^{2} C_{5} .
\end{aligned}
$$


Integrating last integrating over $[0, T]$, we have

$$
\begin{aligned}
& \left\|y_{t}\right\|_{L^{2}\left(0, T ; V^{*}\right)}^{2} \\
\leq & \left(4+4 \lambda^{2} C_{4}\right) \max \{T, 1\}\left(\left\|f+B^{*} \bar{\omega}\right\|_{L^{2}\left(0, T ; V^{*}\right)}^{2}+\|\psi\|_{H}^{2}\right) \\
& +4\left(\varepsilon+C_{2}\right)^{2}\|y\|_{L^{2}(0, T ; V)}^{2}+\left[4\left(C_{2}+\frac{k}{2} C_{3}^{2}\right)^{2}+4 \lambda^{2} C_{5}\right] T \\
\leq & {\left[\left(4+4 \lambda^{2} C_{4}\right) \max \{T, 1\}+4 C_{8}\left(\varepsilon+K_{2}\right)^{2}\right]\left(\left\|f+B^{*} \bar{\omega}\right\|_{L^{2}\left(0, T ; V^{*}\right)}^{2}\right.} \\
& \left.+\|\psi\|_{H}^{2}\right)+4 T\left[\left(C_{2}+\frac{k}{2} C_{3}^{2}\right)^{2}+\lambda^{2} C_{5}\right]+4\left(\varepsilon+C_{2}\right)^{2} \\
= & C_{10}\left(\left\|f+B^{*} \bar{\omega}\right\|_{L^{2}\left(0, T ; V^{*}\right)}^{2}+\|\psi\|_{H}^{2}\right)+C_{11},
\end{aligned}
$$

where

$$
\begin{aligned}
& C_{10}=\left(4+4 \lambda^{2} C_{4}\right) \max \{T, 1\}+4 C_{8}\left(\varepsilon+K_{2}\right)^{2}, \\
& C_{11}=4 T\left[\left(C_{2}+\frac{k}{2} C_{3}^{2}\right)^{2}+\lambda^{2} C_{5}\right]+4\left(\varepsilon+C_{2}\right)^{2}
\end{aligned}
$$

are positive constants.

Combine (2.36) and (2.38), we obtain

$$
\begin{aligned}
\|y\|_{W(0, T ; V)}^{2} & =\|y\|_{L^{2}(0, T ; V)}^{2}+\|y\|_{L^{2}\left(0, T ; V^{*}\right)}^{2} \\
& \leq L_{1}\left(\left\|f+B^{*} \bar{\omega}\right\|_{L^{2}\left(0, T ; V^{*}\right)}^{2}+\|\psi\|_{H}^{2}\right)+L_{2},
\end{aligned}
$$

where $L_{1}=C_{8}+C_{10}, L_{2}=C_{9}+C_{11}$.

This completes the proof of the theorem.

\section{Optimal control problem}

In this section, we discuss the optimal control problem associated with the viscous weakly dispersive BBM equation.

Consider the following control system:

$$
\left\{\begin{array}{l}
\min \{J(y, \bar{\omega})\}=\frac{1}{2}\|C y-z\|_{S}^{2}+\frac{\delta}{2}\|\bar{\omega}\|_{L^{2}\left(Q_{0}\right)}^{2} \\
y_{t}-\varepsilon y_{x x}+\lambda y_{x}+u_{x} y+u_{x}-u_{x} u_{x x}=f+B^{*} \bar{\omega} \\
y(x, 0)=u(x, 0)-u_{x x}(x, 0)=\psi \in H \\
u(0, t)=u(1, t)=u_{x}(0, t)=u_{x}(1, t)=u_{x x}(0, t)=u_{x x}(1, t)=0
\end{array}\right.
$$

where $y=u_{x}-u_{x x}$ and $\bar{\omega}$ is a control in $L^{2}\left(Q_{0}\right), C \in \mathcal{L}(W(0, T ; V), S)$ is a given continuous observation operator, $S$ is a real Hilbert space, and

$$
\min \{J(y, \bar{\omega})\}=\frac{1}{2}\|C y-z\|_{S}^{2}+\frac{\delta}{2}\|\bar{\omega}\|_{L^{2}\left(Q_{0}\right)}^{2},
$$

is performance index of tracking type, here $z \in S$ is desired state and $\delta>0$ is fixed.

The optimal control problem for the viscous weakly dispersive BBM equation is

$$
\min \{J(y, \bar{\omega})\},
$$


where the optimal control pair $(y, \bar{\omega})$ satisfies Eqs.(2.2) with the given initial value and boundary condition.

The following theorem is presented to demonstrate the existence of the optimal control to the viscous weakly dispersive BBM equation.

Theorem 3.1. Let $\psi \in H, f \in L^{2}\left(0, T ; V^{*}\right)$. Then there exists an optimal control $\bar{\omega}$ for the control system (3.1).

Proof. For each $\bar{\omega}$, from Theorem 2.1, there exists a unique weak solution $y$ of Eqs.(2.2). In view of (3.1), we get

$$
J(y, \bar{\omega}) \geq \frac{\delta}{2}\|\bar{\omega}\|_{L^{2}\left(Q_{0}\right)}^{2} \geq 0
$$

for each control pair $(y, \bar{\omega}) \in W(0, T ; V) \times L^{2}\left(Q_{0}\right)$. Thus there exists a constant $\gamma \geq 0$, such that

$$
\gamma=\inf _{W(0, T ; V) \times L^{2}\left(Q_{0}\right)} J(y, \bar{\omega}) .
$$

Choosing the minimizing sequence $\left\{\left(y^{n}, \bar{\omega}^{n}\right)\right\}_{n \in N}$ satisfy Eqs.(2.2), such that

$$
\gamma=\lim _{n \rightarrow+\infty} J\left(y^{n}, \bar{\omega}^{n}\right) \text {. }
$$

From Theorem 2.2, we know that

$$
\|y\|_{W(0, T ; V)} \rightarrow+\infty \Longrightarrow\|\bar{\omega}\|_{L^{2}\left(Q_{0}\right)} \rightarrow+\infty .
$$

So, it follows from (3.2)-(3.4) that $\left\{\left(y^{n}, \bar{\omega}^{n}\right)\right\}_{n \in N}$ is bounded in the Hilbert space $W(0, T ; V) \times L^{2}\left(Q_{0}\right)$, and there exists $\left(y^{*}, \bar{\omega}^{*}\right)$, such that

$$
\begin{cases}y^{n} \rightarrow y^{*}, & \text { weakly in } W(0, T ; V) \\ \bar{\omega}^{n} \rightarrow \bar{\omega}^{*}, & \text { weakly in } L^{2}\left(Q_{0}\right) .\end{cases}
$$

Thus, from (3.5), we have

$$
\lim _{n \rightarrow+\infty} \int_{0}^{T}\left\langle y_{t}^{n}-y^{*}, \varphi(t)\right\rangle_{V^{*}, V} d t=0, \quad \forall \varphi \in L^{2}(0, T ; V) .
$$

Again, from Remark 2.1, we know that $y^{n} \rightarrow y^{*}$ strongly in $C(0, T ; H)$. Furthermore, $u^{n} \rightarrow u^{*}, u_{x}^{n} \rightarrow u_{x}^{*}, u_{x x}^{n} \rightarrow u_{x x}^{*}$ strongly in the space $C(0, T ; H)$. Since $W(0, T ; V)$ is compactly embedded into $L^{2}\left(0, T ; L^{\infty}(\Omega)\right)$ [14], then $y^{n} \rightarrow$ $y^{*}$ strongly in the space $L^{2}\left(0, T ; L^{\infty}(\Omega)\right)$. Moreover, in view of $\left\{y^{n}\right\}$ convergence weakly in $W(0, T ; V)$, then $\left\|y^{*}\right\|_{W(0, T ; V)}$ is bounded, and so is $\left\|y^{*}\right\|_{L^{2}\left(0, T ; L^{\infty}\right)}$.

We remark that the above estimates are sufficient to pass to the limit in the linear terms of Eqs.(2.2). As far as nonlinear term of Eqs.(2.2), by using Holder's inequality, we get

$$
\begin{aligned}
& \left|\int_{0}^{T}\left(u_{x}^{n} y^{n}-u_{x}^{*} y^{*}, \varphi\right)_{H} d t\right| \\
\leq & \left|\int_{0}^{T}\left(u_{x}^{n}\left(y^{n}-y^{*}\right), \varphi\right)_{H} d t\right|+\left|\int_{0}^{T}\left(\left(u_{x}^{n}-u_{x}^{*}\right) y^{*}, \varphi\right)_{H} d t\right|
\end{aligned}
$$




$$
\begin{aligned}
& \leq \int_{0}^{T}\left\|u^{n}\right\|_{H}\|\varphi\|_{V}\left\|y^{n}-y^{*}\right\|_{L^{\infty}} d t+\int_{0}^{T}\left\|u^{n}-u^{*}\right\|_{H}\|\varphi\|_{V} \|_{y^{*} \|_{L^{\infty}} d t} \\
& \leq\left\|u^{n}\right\|_{C(0, T ; H)}\|\varphi\|_{L^{2}(0, T ; V)}\left\|y^{n}-y^{*}\right\|_{L^{2}\left(0, T ; L^{\infty}\right)} \\
& \quad+\left\|y^{*}\right\|_{L^{2}\left(0, T ; L^{\infty}\right)}\|\varphi\|_{L^{2}(0, T ; V)}\left\|u^{n}-u^{*}\right\|_{C(0, T ; H)} \\
& \longrightarrow 0, \quad n \rightarrow+\infty, \forall \varphi \in L^{2}(0, T ; V), \\
&\left|\int_{0}^{T}\left(u_{x}^{n} u_{x x}^{n}-u_{x}^{*} u_{x x}^{*}, \varphi\right)_{H} d t\right|=\frac{1}{2}\left|\int_{0}^{T}\left(\left(u_{x}^{n}\right)^{2}-\left(u_{x}^{*}\right)^{2}, \varphi\right)_{H} d t\right| \\
& \leq \frac{1}{2}\left|\int_{0}^{T}\left\|\left(u_{x}^{n}\right)^{2}-\left(u_{x}^{*}\right)^{2}\right\|_{H}\|\varphi\|_{H} d t\right| \\
& \leq \frac{1}{2} T^{\frac{1}{2}}\left\|\left(u_{x}^{n}\right)^{2}-\left(u_{x}^{*}\right)^{2}\right\|_{C(0, T ; H)}\|\varphi\|_{L^{2}(0, T ; H)} \\
& \longrightarrow 0, n \rightarrow+\infty, \forall \varphi \in L^{2}(0, T ; V) .
\end{aligned}
$$

From (3.5), we know that

$$
\int_{0}^{T} \int_{0}^{1}\left(B^{*} \bar{\omega}^{n}-B^{*} \bar{\omega}^{*}\right) \varphi d x d t \rightarrow 0, \quad n \rightarrow+\infty, \forall \varphi \in L^{2}(0, T ; V) .
$$

Since $y^{n} \rightarrow y^{*}$ strongly in $C(0, T ; H)$, we can infer that $y^{n}(0) \rightarrow y^{*}(0)$ weakly in $H$ as $n \rightarrow+\infty$. Then, we have

$$
y^{*}(0)=\psi .
$$

From the analysis above, we deduce that $\left(y^{*}, \bar{\omega}^{*}\right)$ satisfies Eqs.(2.2), and such that

$$
J\left(y^{*}, \bar{\omega}^{*}\right)=\min \{J(y, \bar{\omega})\} .
$$

Hence, there exists an optimal control to the control system (3.1).

Remark 3.1. In view of the relation $u=\left(1-\partial_{x}^{2}\right)^{-1} y$, there exists an optimal control $\left(u^{*}, \bar{\omega}^{*}\right)$ for the viscous weakly dispersive BBM equation (2.1).

\section{References}

[1] J. Avrin and J. A. Goldstein, Global existence for the Benjamin-Bona-Mahony equation in arbitrary dimensions, Nonlinear Anal. 9 (1985), no. 8, 861-865.

[2] T. B. Benjamin, J. L. Bona, and J. J. Mahony, Model equations for long waves in nonlinear dispersive systems, Philos. Trans. Roy. Soc. London Ser. A. 272 (1972), no. 1220, 47-78.

[3] H. Chen, Periodic initial value problem for BBM equation, Comput. Math. Appl. 48 (2004), no. 9, 1305-1318.

[4] D. J. Korteweg and G. de Vries, On the change of form of long waves advancing in a rectangular canal, and on a new type of long stationary waves, Philos. Magazine 39 (1895), 422-443.

[5] J. Lenells and M. Wunsch, On the weakly dissipative Camassa-Holm, DegasperisProcesi, and Novikov equations, J. Differential Equations 255 (2013), no. 3, 441-448.

[6] J. L. Lions, Optimal Control of Systems Governed by Partial Differential Equations, Springer, Berlin, 1971. 
[7] S. Micu, On the controllability of the linearized Benjamin-Bona-Mahony equation, SIAM J. Control Optim. 39 (2001), no. 6, 1677-1696.

[8] J. Nickel, Elliptic solutions to a generalized BBM equation, Phys. Lett. A. 364 (2007), no. 3-4, 221-226

[9] L. Rosier and B. Zhang, Unique continuation property and control for the BenjaminBona-Mahony equation on a periodic domain, J. Differential Equations 254 (2013), no. $1,141-178$.

[10] S. U. Ryu and A. Yagi, Optimal control of Keller-Segel equations, J. Math. Anal. Appl. 256 (2001), no. 1, 45-66.

[11] C. Shen and A. Gao, Optimal control of the viscous weakly dispersive Degasperis-Procesi equation, Nonlinear Anal. 72 (2010), no. 2, 933-945.

[12] N. Smaoui, Boundary and distributed control of the viscous Burgers equation, J. Comput. Appl. Math. 182 (2005), no. 1, 91-104.

[13] B. Sun, Maximum principle for optimal distributed control of the viscous DullinGottwald-Holm equation, Nonlinear Anal. 13 (2012), no. 1, 325-332.

[14] R. Temam, Navier-Stokes Equations: Theory and Numerical Analysis, North-Holland Pub. Co, 1979.

[15] L. Tian and C. Shen, Optimal control of the viscous Degasperis-Procesi equation, J. Math. Phys. 48 (2007), no. 11, 113513, 16 pp.

[16] L. Tian, C. Shen, and D. Ding, Optimal control of the viscous Camassa-Holm equation, Nonlinear Anal. Real World Appl. 10 (2009), no. 1, 519-530.

[17] R. Vedantham, Optimal control of the viscous Burgers equation using an equivalent index method, J. Global Optim. 18 (2000), no. 3, 255-263.

[18] L. Zeng, Existence and stability of solitary wave solutions of equations of BenjaminBona-Mahony type, J. Differential Equations 188 (2003), no. 1, 1-32.

[19] S. Zheng, Nonlinear Evolution Equations, CRC Press, 2004.

\section{LEI ZHANG}

School of Mathematics and Statistics

Huazhong University of SCIEnCE AND TeChNology

Wuhan 430074, Hubei, P. R. China

E-mail address: 850887042@qq.com

BIN LIU

School of Mathematics and Statistics

Huazhong University of Science and Technology

Wuhan 430074, Hubei, P. R. China

E-mail address: binliu@mail.hust.edu.cn 